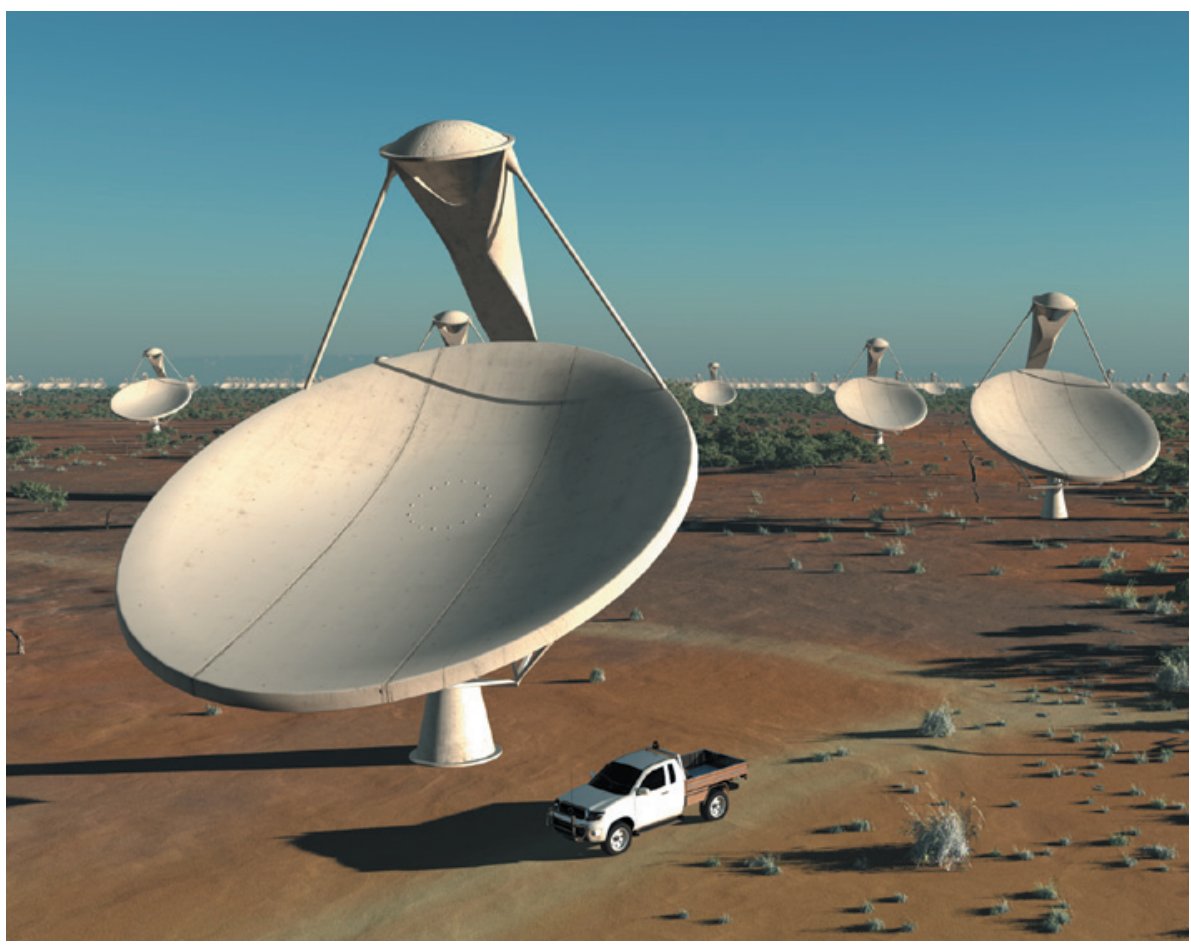

The Square Kilometre Array would be the world's most powerful radio telescope (artist's impression).

ASTRONOMY

\title{
Giant telescope may get two homes
}

\section{Split-site solution could allow both Australia and South Africa to host parts of the Square Kilometre Array.}

\section{BY GEOFF BRUMFIEL}

$\mathrm{W}$ ith the battle to host the world's most powerful radio telescope growing increasingly acrimonious, the project's leaders are considering whether to divide the spoils.

The US\$2.1-billion Square Kilometre Array (SKA) would open a window on the early Universe. As yet, international partners have not committed to covering the hefty price tag. But if the project goes ahead, it would bring a flood of funding, prestige and scientific opportunities to one of the two competing teams: South Africa or joint bidders Australia and New Zealand.

Last month, after considering the scientific merits of the two sites, the SKA Site Advisory Committee concluded that South Africa offered marginally better opportunities (see Nature http://doi.org/hst; 2012). Since then, the already-intense lobbying from both sides has increased, with politicians from each country insisting that they would prevail.
Now, the SKA management board has asked a new scientific panel to determine whether the telescope, made up of 3,000 15-metrewide dish antennas and many more simpler antennas, could be divided between the two proposed sites.

Politicians in Australia and South Africa say that they oppose any split in the project, but John Womersley, head of the SKA board and chief executive of the UK Science and Technology Facilities Council, says that a compromise may be one way to resolve the battle. "I have heard astronomers that I respect say that such a solution is possible," Womersley told Nature.

Astronomers plan to use the SKA to measure radio signals at a frequency of around 1 gigahertz, probing early galaxy formation and investigating how gravity behaves near black holes. Capable of detecting a television के signal from up to 15 parsecs (50 light years) away, the telescope might even aid in the search for extraterrestrial intelligence. Supercomputers will integrate the signals from the dishes and antennas, creating a virtual dish with a combined collecting area of 1 square kilometre (see Nature 480, 308-309; 2011).

"Normally when you have a giant dish, you cannot split it, but the SKA has many different components," says Heino Falcke, a radio astronomer at Radboud University in Nijmegen, the Netherlands. The easiest solution would be to divide the project by placing the higher-frequency dishes on one continent and the lowerfrequency antennas on the other.

Doing so would almost certainly raise the SKA's price tag, because computing centres and power would be needed in both remote locations, says Albert Zijlstra, director of the Jodrell Bank Centre for Astrophysics in Manchester, UK. But splitting the antennas by frequency would avoid the need for a high-throughput data link connecting the two sites, something that Zijlstra and Falcke both expect would be even more costly.

Zijlstra says that he can see little scientific advantage to splitting the project, aside from a few extra hours of observation time gained from having the telescope's two parts separated by such a vast distance. Falcke agrees. "It's a question of politics," he says.

The panel is expected to deliver its results by mid-May, when the SKA board will meet again to discuss the site.

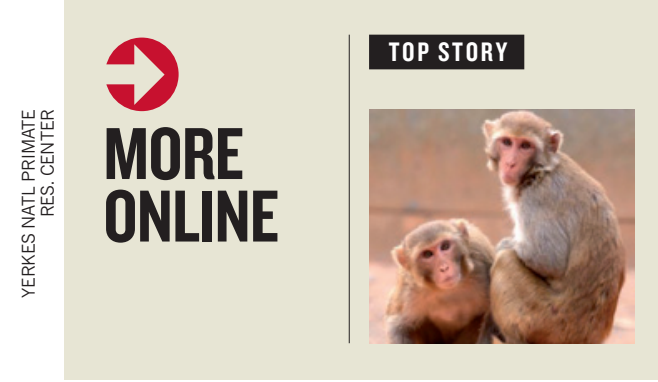

Social status changes gene expression in monkeys go.nature.com/ mkjjbo

\section{MORE NEWS}

- Magnetic storms spotted on Venus go.nature.com/14wqgw

- Astronomers find solar system more crowded than ours go.nature.com/ s4ejqr

- United Nations to appoint a chief scientific adviser go.nature.com/tnk229

\section{Q\&A}

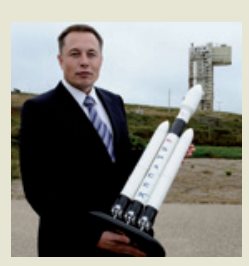

Privatespaceflight pioneer Elon Musk sets his sights on Mars go.nature.com/ w99pls 